\section{Donepezil - QT prolongation and torsades de pointes}

Editor - I read with interest, the 'Lesson of the month 1: Prolonged QT syndrome due to donepezil: a reversible cause of falls?' by Emily G Jackson and Sarah Stowe. ${ }^{1}$ Being a higher specialist trainee in geriatric medicine, I have seen a few cases of cardiac conduction disorders arising from the use of cholinesterase inhibitors such as donepezil, galantamine and rivastigmine in patients with cognitive decline. ${ }^{2}$ I will like to bring your readers' attention to the fact that although British National Formulary (BNF) does not specifically mention QT prolongation and torsades de pointes as listed side effect of donepezil, it does state that 'cardiac conduction disorders' are a rare or very rare side effect of donepezil, so care and caution should be exercised in interpreting this fact. ${ }^{3}$ I also wanted to highlight some facts regarding CredibleMeds.org, published on their own website. It is a federally funded, university-based centre for education and research on therapeutics founded in 1999. It is used as a resource by some individual healthcare professionals registered with their website, a few hospitals including the Mayo Clinic in Rochester, Minnesota and New Zealand's federal Medicines Safety Authority for the safety of anti-depressants. ${ }^{4}$ But I am yet to find a source that confirms its recognition by the National Institute for Health and Care Excellence, the BNF or any of the UK based NHS trusts, therefore information offered by this website should be interpreted with caution by the healthcare professionals in the United Kingdom.

BILAL HAIDER MALIK ST4 geriatric medicine and general internal medicine, Colchester, UK

\section{References}

1 Jackson EG, Stowe S. Lesson of the month 1: Prolonged QT syndrome due to donepezil: a reversible cause of falls? Clin Med 2019;19:80-1.

2 Alzheimer's disease: beware of interactions with cholinesterase inhibitors. Prescrire Int 2006;15:103-6.

3 British National Formulary. Donepezil hydrochloride. British National Formulary, 76. BNF, 2018. https://bnf.nice.org.uk/drug/donepezilhydrochloride.htm|\#sideEffect.

4 About CredibleMeds. CredibleMeds. https://crediblemeds.org/index. php?cID $=222$.

\section{Care of the older surgical patient}

Editor - We welcome the paper exploring the role of the geriatrician in the care of the older surgical patient. ${ }^{1}$ This paper highlights the difficulties involved in developing new models of care in the perioperative setting. Despite the positive points raised by the paper, we feel the authors have missed a significant opportunity relating to the pre-optimisation of these complex patients. In the care pathways presented, the input of a geriatrician at the proposed points is far too late in the process. Optimal outcomes would be most likely if a geriatrician is involved early in the pre-assessment and optimisation phase. This model of care is currently being championed by the Proactive Care of Older People team at Guy's and St Thomas' Hospital. We have seen that this model of care, when applied, can produce a significant improvement in outcomes. ${ }^{2}$ There is growing evidence in the literature that comprehensive geriatric assessment and optimisation in the pre-operative phase can lead to improved outcomes postoperatively. ${ }^{3}$ While improvements have been achieved by the involvement of geriatricians later in the process, this is not what we should aspire to when designing new care pathways.

The study does propose interesting questions with regard to who will provide this extra 'expertise' to obtain the very best clinical outcomes for these patients. Geriatricians are well placed to help provide this care, although simply using geriatricians without training in this area of medicine would not be the optimal solution. We have to recognise that all the specialties involved require training in perioperative medicine. This has been a major area of interest in anaesthesia over the last few years with further training being included in our curriculum. The Royal College of Anaesthetists have recently endorsed a University College London e-learning course to help with this process. ${ }^{4}$ Integrated cross specialty training programmes are required to deliver this training in the future.

In our opinion, there should not be one individual specialty seeking to provide this care but ideally all specialties involved working together in an multidisciplinary team approach throughout the patients perioperative pathway. As perioperative medicine develops, input from anaesthetists will hopefully become increasingly present in these new care pathways, especially in the postoperative environment. ${ }^{5}$ As a specialty we are often heavily involved in the planning and delivery of care to these patients and would be ideally placed to provide support postoperatively.

Whilse practically we appreciate that this is difficult to achieve, it must be the foundation on which we continue to re-design and build new models of future care in this area. Complex older surgical patients require cross specialty input to make significant improvements in the health outcomes of this high-risk surgical group.

SIMON TRUNDLE

ST7 in anaesthesia and intensive care medicine, Royal London Hospital, Bart's Health NHS Trust, London, UK

HELEN DREWERY

Consultant anaesthetist, Royal London Hospital, Bart's Health NHS Trust, London, UK

\section{References}

1 Howie S, Tinker A. Are we on the same page? Exploring the role of the geriatrician in the care of the older surgical patient from the perspective of surgeons and geriatricians. Clin Med 2018;18:374-9.

2 Partridge JS, Harari D, Martin FC et al. Randomised clinical trial of comprehensive geriatric assessment and optimisation in vascular surgery. Br J Surg 2017; 104:680.

3 Partridge JS, Harari D, Martin FC, Dhesi JK. The impact of preoperative comprehensive geriatric assessment on postoperative outcomes in older patients undergoing scheduled surgery: a systematic review. Anaesthesia 2014;69(Suppl 1):9.

4 Future Learn. Perioperative medicine in action: e-learning course. Future Learn. https://www.futurelearn.com/courses/perioperativemedicine [Accessed 17 January 2019].

5 Grocott MPW, Pearse RM. Perioperative medicine: the future of anaesthesia? Br J Anaesth 2012;108:723-6. 